\title{
Maxillary osteosarcoma: Morphological variants, clinicopathological picture, and literary revision
}

\author{
Avissai Alcántara-Vázquez ${ }^{1,2 *}$, Mercedes Hernández-González ${ }^{1,2}$, Daniel López-Zuñiga ${ }^{1,2}$, and \\ Elliette Alcántara-Burguete ${ }^{3}$ \\ ${ }^{1}$ Surgical Pathology Unit, Hospital General de México "Dr Eduardo Liceaga"; ${ }^{2}$ School of Medicine, Universidad Autónoma de México (UNAM); \\ ${ }^{3}$ Oncology Unit, Hospital General de México "Dr. Eduardo Liceaga”. Mexico City, Mexico
}

\begin{abstract}
Objective: Jaw osteosarcoma shows a variety of histomorphological clinical conditions. Despite the advances in molecular pathogenesis and biological markers, clinicopathological correlation is considered the most important criterion in diagnosis. Material and methods: The database of the Surgical Pathological Department of the General Hospital of Mexico was reviewed to obtain cases of osteosarcoma during the period from 2002 to 2019 . The cases were evaluated on the Enneking scale, which has prognostic considerations. Results: Regarding the 11 cases found, $54.5 \%$ are women. The average age was 36 years old, the primary location was the jaw. The average size was $8 \mathrm{~cm}$. Clinicopathological concordance was $27.3 \%$. The most frequent histologic variant was chondroblastic. The predominant histological grade was high and $83 \%$ of the cases had surgical margins involved. Conclusion: The patients were in the fourth decade of their life, the tumors were large in size with a high histological grade of malignity and the majority of cases had affected surgical margins. All data had a bad prognostic.
\end{abstract}

Key words: Osteosarcoma. Jaws. Histological type. Stage.

\section{Introduction}

Osteosarcoma (OS) is a primary malignant tumor originating from primitive osteoid-producing primitive mesenchymal cells and disorganized immature bone.

Jaw osteosarcoma (JOS) is rare, occurring in about $6 \%$ of $\mathrm{OS}^{1,2}$. It appears two decades later than long bone OS, at an average age between 33 and 36 years $^{3}$. The risk of developing metastases is between $20 \%$ and $25 \%$, whereas long bone OS is between $44 \%$ and $49 \%^{2}$. The average survival rate is better at $77 \%$ at 5 years, when the tumor is localized and the resection is complete ${ }^{4}$.
According to the WHO classification of OS, it can be classified as high grade, intermediate, and low grade. Most JOS are high grade and include the conventional type, comprising osteoblastic, chondroblastic, and fibroblastic forms, and other rare varieties such as giant cell-rich, well-differentiated fibroblastic, epithelioid, and small cell ${ }^{5}$, depending on the amount of osteoid, cartilage, or collagen produced by the tumor cells.

The etiology of OS remains unknown. There is an absence of consistent molecular alterations and the karyotype is often complex 6 . The tumor suppressor genes p53 and Rb1 are frequently mutated and appear
Available online: 29-07-2021 Rev Med Hosp Gen Mex. 2021;84(3):104-109 www.hospitalgeneral.mx CC BY-NC-ND license (http://creativecommons.org/licenses/by-nc-nd/4.0/). 
to be involved in the onset of the disease ${ }^{7}$. It has also been established that the bone environment plays an important role in the development, progression, and chemoresistance of $\mathrm{OS}^{8}$. The difference in the clinical and biological behavior of long bone OS and JOS is due to the different microenvironment between the two sites. The major alterations involve p53 and RB1 with $80 \%$ $90 \%$ and $10 \%-39 \%$, respectively, and to a lesser extent, ATRX, Dbl2, RUNSX2, and PTEN ${ }^{9}$ genes are affected.

Alterations in p16 protein expression correlate with the pathogenesis and progression of $\mathrm{OS}^{9}$ and there is a correlation between p16 negativity and the risk of an unfavorable outcome of $\mathrm{OS}^{10}$.

Immune infiltrates form the key component of the complex local environment of OS ${ }^{11}$. This microenvironment produces everything necessary for the control of proliferation, drug resistance, and cell dissemination ${ }^{12}$. Macrophages are the major representative of the immune infiltrate and tumor-associated macrophages control local immunity, angiogenesis, and regulate tumor cell migration ${ }^{13}$. The immune profile of JOS has been explored by immunohistochemistry, finding low levels of CD4 and CD8, as well as low numbers of T-lymphocytes associated with antigen 4 and programmed cell death protein. However, no association was found between the immune profile and clinicopathological findings ${ }^{14}$.

In angiogenesis, Jawad et al..$^{15}$ compared the vascular endothelial growth factor response in long bone OS and JOS, finding less expression in the latter, which could explain its low metastatic potential and poor response to neoadjuvant therapy.

JOS can be part of some hereditary syndromes such as hereditary retinoblastoma, Li-Fraumeni syndrome, Paget's disease of bone, Rothmund-Thomson syndrome, and Werner syndrome or as sporadic JOS, by far the most frequent form ${ }^{16}$.

The maxilla and mandible are affected with equal frequency; the tumor originates in the posterior part of the body of the mandible and in the horizontal branch rather than in the ascending branch. Lesions of the maxilla are located in the alveolar ridge, floor of the maxillary sinus, and palate rather than in the zygoma and orbital ring ${ }^{17}$. The most common presenting symptoms are enlargement and pain. Other features include facial deformity, tooth loss, paresthesia, toothache, bleeding, and nasal obstruction. JOS most commonly affects males ${ }^{18}$.

Radiologically, JOS may present with sclerotic or radiolucent lesions; the classic "sunburst" appearance is due to periosteal bone production. The other classic image is due to infiltration of the tumor along the periodontal ligament, enlarging the periodontal space called "Garrington's sign"17. The diagnosis of JOS is based on radiological findings and cell morphology data. As for immunohistochemistry, attempts have been made to separate the various histological subtypes. Thus, osteonectin and osteocalcin have been widely used. Osteocalcin is specific for osteoblasts, while osteonectin is positive in other cells such as fibroblasts, endothelial cells, chondrocytes, nerves, and giant cells. Other markers are S-100 protein, SOX9, Ki67, Bcl2, p53, caveolin-1, and CD99. However, due to the heterogeneity of tumors, a conclusive profiling of histological subtypes has not been achieved, and therefore, histological assessment is still considered the "gold standard"19,20.

The classification of OS is based on their location and histological presentation. According to their location, they are divided into central, paraosteal, and periosteal. Histologically speaking, the main feature is the presence of the fundamental cell which is the osteoblast and malignant osteoid in the stroma. In addition, seven cell types have been reported, namely, chondroblast-like cells, fibroblast, histiocytes, myofibroblasts, osteoclasts, and angioblasts. Depending on the cell present and the type of matrix, OS is divided into osteoblastic, chondroblastic, fibroblastic, telangiectatic, low-grade fibroblastic, giant cell-rich, epithelioid, and small cell types ${ }^{18}$.

The prognosis of OS is usually determined by the Enneking system ${ }^{21}$ which evaluates the histological grade $(G)$, the extension of the primary tumor $(T)$ as intra- or extra-compartmental, as well as metastases to regional lymph nodes or other organs (M).

Among the histological subtypes, the chondroblastic variety is the most resistant to treatment and therefore has an adverse prognosis. The fibroblastic type responds to treatment and has a better prognosis ${ }^{22}$. The main prognostic criteria in JOS are tumor size and complete resection ${ }^{23}$. Complete resection is mainly difficult in the maxilla, therefore, local recurrence rather than metastasis is most frequent, consequently positive surgical margins are associated with poor prognosis ${ }^{24}$.

JOS is relatively radioresistant. Hence, high doses are used. Chemotherapy improves survival in non-metastatic long spindle OS. However, neoadjuvant chemotherapy helps by improving local control and decreasing the incidence of pulmonary metastases ${ }^{18}$.

JOS is a rare tumor of the oral region and an understanding of the histological aspect is required, reducing diagnostic difficulties by separating these tumors from benign diseases of these bones. 


\section{Materials and methods}

A search for JOS was carried out in the archives of the Surgical Pathology Unit of the Hospital General de México, during the period 2002-2019. The following was obtained from the cases found: histopathological reports, clinical data, slides, and paraffin slides for complementary sections if necessary. Two surgical pathologists reviewed all histological material with light microscopy. For histological grading, the Broder's classification system ${ }^{25}$ was used, which is based on the degree of cellularity, pleomorphism, mitotic activity, evidence of invasion, and necrosis, resulting in four grades of malignancy.

For specific grading details in bone neoplasms, the Unni classification ${ }^{26}$ was used. It grades tumors as lowgrade malignancy or G1 if they are classified as Broder's Grades 1 and 2 and high grade or G2 if they are classified as 3 or 4 . Finally, these data were organized into stages according to the Enneking classification ${ }^{21}$, which has prognostic purposes.

\section{Results}

Eleven cases of JOS were found, of which 6 (54.5\%) came from surgical specimens, $3(27.3 \%)$ from slides and paraffin blocks, and 2 cases (18.2\%) from biopsies.

The specific clinical diagnosis of JOS was made in 3 cases (27.3\%), tumor only was diagnosed in 3 cases $(27.3 \%)$, and with 1 case $(9.1 \%)$. Diagnoses of nasofibroma, giant cell granuloma, osteochondroma, carcinoma, and granulomatous sialoadenitis were also made.

The tumors were most frequently found in women, 6 cases $(54.5 \%)$ versus 5 cases $(45.5 \%)$ in men. The average age was 36 years, with limits ranging from 5 to 65 years, $54.6 \%$ of the cases were found between 20 and 40 years of age. The most frequent location was the maxilla with $54.5 \%$ and $45.5 \%$ in the mandible, in both bones mainly on the left side.

The size of the tumor could only be determined in six cases, the smallest being $2 \mathrm{~cm}$, the largest $15 \mathrm{~cm}$, and the average $8 \mathrm{~cm}$. In terms of bone location, all $11 \mathrm{tu}$ mors were central, none were paraosteal. Macroscopically, JOS are tumors with poorly defined boundaries, yellowish-gray color, sandy surface, firm consistency with chondroid, hemorrhagic, or necrotic areas (Fig. 1).

Histologically speaking, the tumor is made up of malignant, spindle-shaped mesenchymal malignant cells that produce osteoid or immature bone that infiltrates

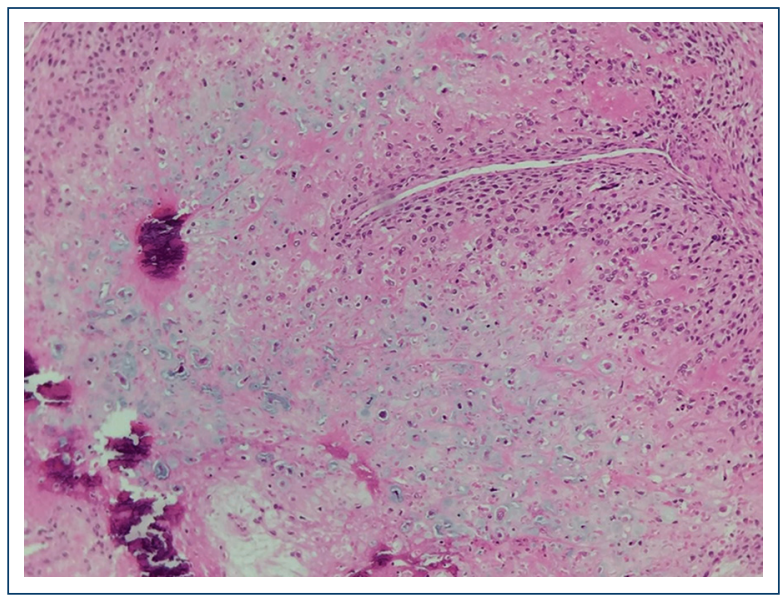

Figure 1. Chondroblastic osteosarcoma. Tumor with a stroma of pleomorphic cells, which form osteoid and undergo chondromatous transformation $(\times 300)$.

pre-existing bone trabeculae. They are morphologically classified according to their extracellular matrix. Regarding the 11 cases, $36.4 \%$ corresponded to the chondroblastic variety (Fig. 2), $27.2 \%$ to the osteoblastic variety (Fig. 3), and $9.1 \%$ to the following four varieties: well-differentiated fibroblastic (Fig. 4), giant cell rich (Fig. 5), epithelioid type (Fig. 6), and small cell JOS.

For histological grade, the Broder's classification ${ }^{25}$ was followed. Five cases (45.5\%) were found to be Grade 2 or low grade (G1) in the Unni ${ }^{26}$ classification and 6 cases (54.5\%) were found to be Grade 3 or highgrade malignancy (G2). Staging followed the Enneking system $^{21}$, which comprises three stages, 9 of the 11 cases could be included as two cases corresponded to biopsies. As for nine cases, three were low-grade malignant (G1) and intracompartmental (T1), thus corresponding to Stage IA (G1,T1). Another case was also low grade (G1) but extracompartmental and corresponded to Stage IB (G1,T2). The remaining five cases were of high-grade malignancy (G2), two intracompartmental cases (T1), Stage IIA (G2,T1), and three extracompartmental cases (T2) Stage IIB (G2,T2) (Table 1).

As for six of the 11 cases, it was possible to determine the condition of the surgical edges: 5 cases (83.3\%) had tumor edges and 1 case (16.7\%), with a history of radiotherapy, had no tumor edges.

\section{Discussion}

OSs are malignant bone tumors characterized by the formation of osteoid and immature bone from tumor mesenchymal cells ${ }^{27}$. 


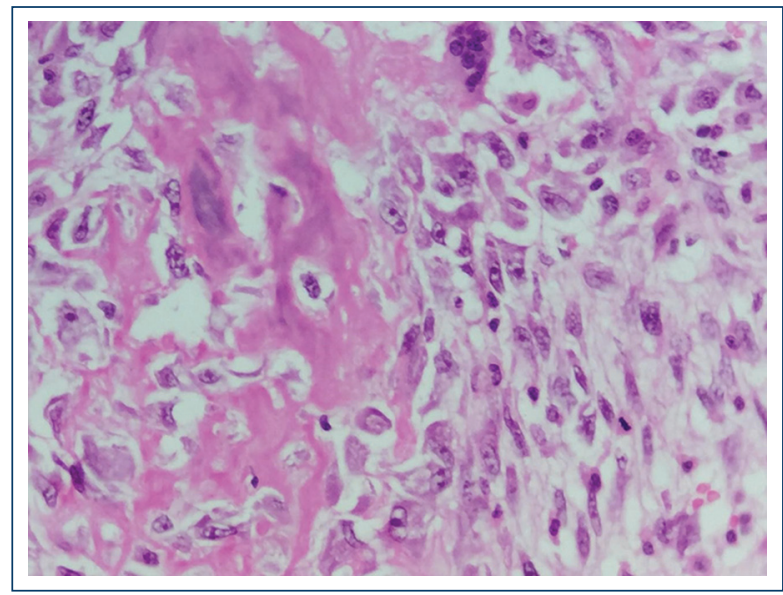

Figure 2. Osteoblastic osteosarcoma shows sarcomatous, pleomorphic stroma with calcified and partially ossified osteoid formation, which is invaded by tumor osteoblasts $(\times 300)$.

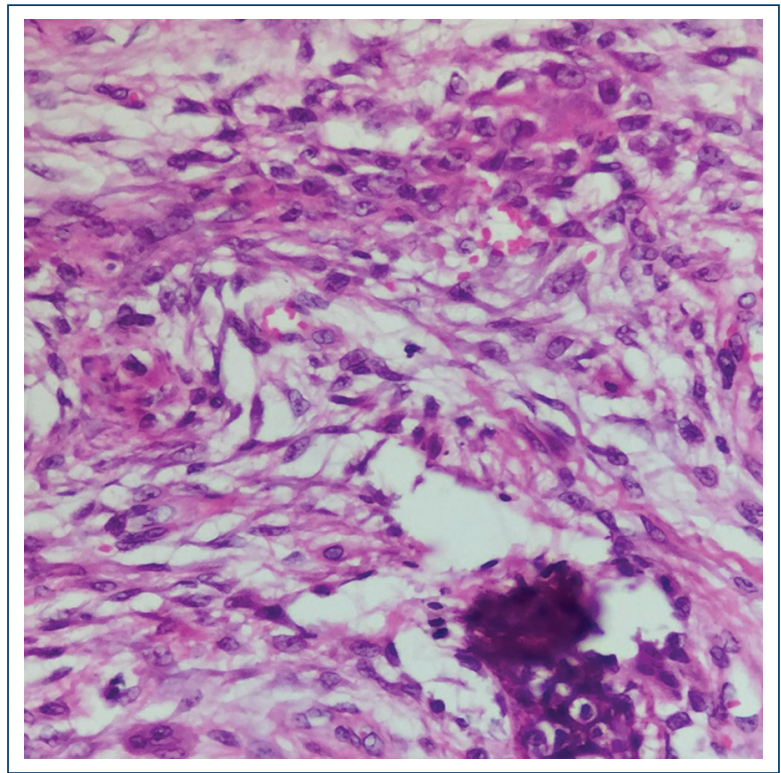

Figure 3. Well-differentiated fibroblastic osteosarcoma; well-differentiated spindle cell sarcomatous stroma, which forms osteoid ( $\times 200)$.

About $10 \%$ of OS occur in the head and neck, most of them in the mandible or maxilla ${ }^{28}$, with an average age between 33 and 36 years $^{3}$. We found an average age of 36 years and Delgado et al. ${ }^{29}$ reported 28 years of age. With regard to sex, we found that the tumor was more frequent in women, which is consistent with the findings of Delgado et al. ${ }^{29}$ With regard to size, Delgado ${ }^{29}$ reported an average of $10 \mathrm{~cm}$ and we found $8 \mathrm{~cm}$.

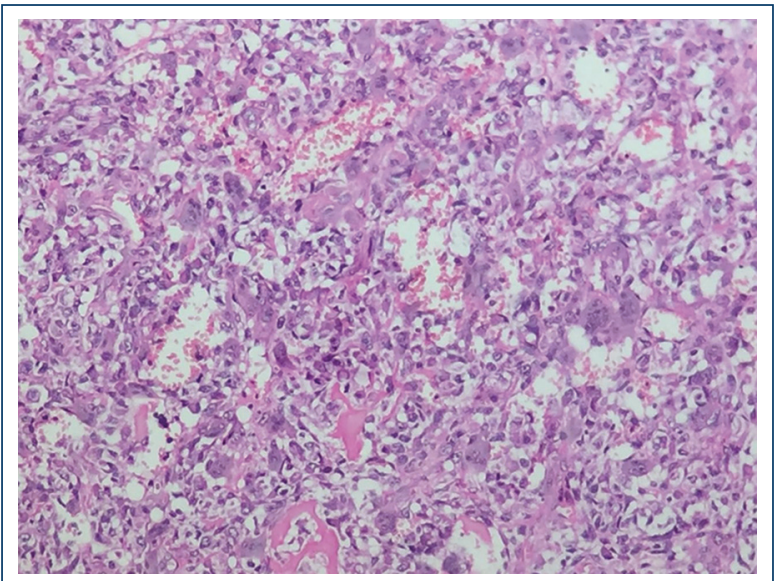

Figure 4. Osteosarcoma rich in giant cells. Sarcomatous stroma with osteoid formation surrounded by abundant multinucleated osteoclastic giant cells $(\times 200)$.

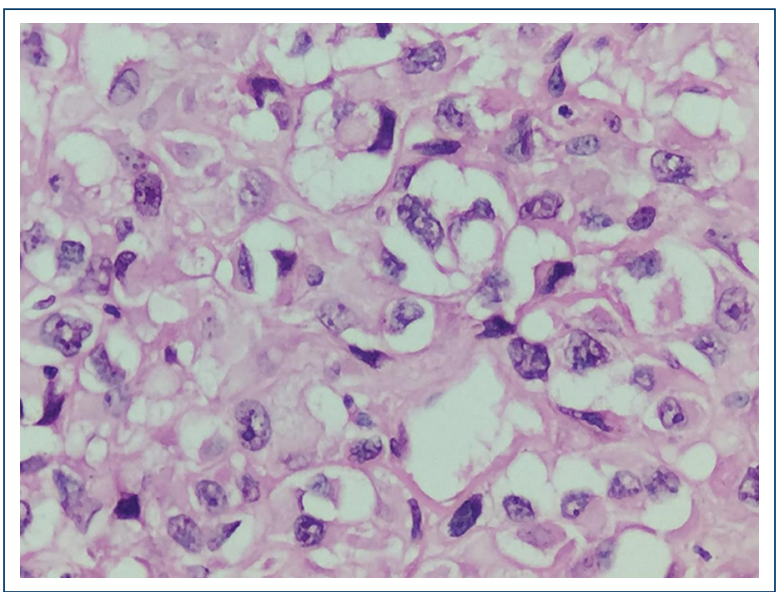

Figure 5. Epithelioid osteosarcoma. Sarcoma consisting of epithelioid osteoblasts which form osteoid in small numbers $(\times 400)$.

Kassir et al. ${ }^{30}$ pointed out that JOS occurs with equal frequency in the mandible and maxilla and has a similar prognosis, but we found that the maxillary bone is the most affected. Clinically speaking, the most frequent symptoms of JOS are enlargement and pain, which occur in $79 \%$ of cases, but establishing the correct diagnosis is not an easy task. Regarding the cases under study, the diagnosis of JOS was made clinically in only $27.3 \%$ of cases. In dental radiographs, two images are indicative of the diagnosis, one is the "sunray" and the other is the "Garrington sign"17. In our series, it was not possible to obtain the radiological signs. Metastases are rare, occurring in 
Table 1. Classification by Enneckin System in 9 cases

\begin{tabular}{|l|c|c|c|c|c|c|c|}
\hline Stage & Number of cases & Grade & Number of cases & Compartment & Number of cases & Metastasis & Number of cases \\
\hline I A & 3 & G1 & 3 & T1 & 3 & M0 & 3 \\
\hline I B & 1 & G1 & 1 & T2 & 1 & M0 & 1 \\
\hline II A & 2 & G2 & 2 & T1 & 2 & M0 & 2 \\
\hline II B & 3 & G2 & 3 & T2 & 3 & M0 & 3 \\
\hline Total & 9 & & 9 & & 9 & & 9 \\
\hline
\end{tabular}

*Two of the 11 cases were biopsies.

G1: Low grade. G2: High grade. T1: Intracompartmental. T2: Extracompartmental.

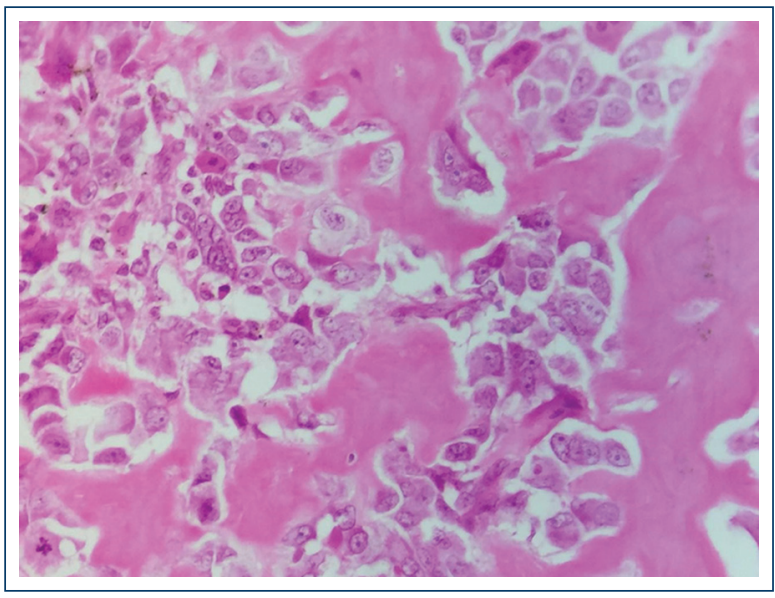

Figure 6. Small cell osteosarcoma. Tumor consisting of lymphoid-like cells, which form a small amount of osteoid material. (× 200).

$5 \%$ of cases, and the lungs are the most affected ${ }^{31}$. JOS is divided into central and peripheral by location; our cases were central or intramedullary. In terms of behavior, they are divided into high-grade and lowgrade malignant, $54.5 \%$ of our cases were high grade and $55.6 \%$ were intracompartmental. Histologically speaking, the conventional type of JOS is the most frequent with $36.4 \%$ of the chondroblastic variety ${ }^{27}$. In our series, in addition to the seven cases of conventional JOS, we found another four cases of rare JOS, such as well-differentiated fibrous JOS, which has a better prognosis than conventional $\mathrm{JOS}^{31,32}$, giant cellrich JOS, and epithelioid $\mathrm{JOS}^{33,34}$ that have a similar prognosis to conventional JOS. In addition, we found one case of small cell JOS, which is more aggressive than conventional $\mathrm{JOS}^{35}$.

In terms of histological grade, $54.5 \%$ of the cases were within the high grade of malignancy, which is similar to that reported by Delgado ${ }^{29}$. In terms of staging according to the Enneking classification ${ }^{21}, 55.5 \%$ were high-grade neoplasms and $44.4 \%$ had extracompartmental location (Table 1).

The prognosis of JOS depends mainly on two criteria: the size of the tumor and complete resection ${ }^{23}$, the latter being difficult to achieve due to the anatomical features of the region, mainly in the maxilla, which results in a poor prognosis ${ }^{24}$. In our cases, complete removal of the tumor was achieved in $16.7 \%$ of cases, lower than Delgado's case report ${ }^{29}$, which was $43 \%$, and the 5-year survival rate in the same author's cases was $10 \%$. Follow-up was not possible in our cases.

Death is secondary to local extension with vascular and neural infiltration ${ }^{31}$. Therefore, even though these tumors are rare, it is necessary to recognize them to detect them in time and be able to remove them completely to achieve prolonged survival or even cure.

This paper combines two important facts, the histomorphological varieties of JOS, the conventional variety and rare varieties, which have prognostic implications, as well as the staging according to Enneking's criteria, which also have prognostic significance. We did not find these facts referred to in the literature.

\section{Conflicts of interest}

The authors declare that there are no conflicts of interest.

\section{Ethical disclosures}

Protection of human and animal subjects. The authors declare that no experiments were performed on humans or animals for this study.

Confidentiality of data. The authors declare that they have followed the protocols of their work center on the publication of patient data. 
Right to privacy and informed consent. The authors declare that no patient data appear in this article.

\section{References}

1. Amrollahie A, Mai TP, Portnof J, Kaltman SI. Primary intramedular osteosarcoma of the maxilla in a pediatric patiente: a case report and review of the literatura. Clin Surg. 2018;3:2022-5.

2. Boom WT, Van der Graaf H, Gerderblom ME, Tesselaar ME, van Es RJ, Oosting SF, et al. Impacta of chemotherapy in the outcome of osteosarcoma of the head and neck in adults. Head Neck. 2017;39:140-6.

3. Kontio R, Hastrom J, Lindholm P, Böhling T, Sampo M, Mesimäki K, et al Craniomaxillofacial osteosarcoma the rol surgical margins. J CraniomaxiIlofac Surg 2019;47:922-5

4. Thariat J, Schouman T, Brouchet A, Sarini J, Miller RC, Reychler H, et al. Osteosarcoma of the mandible: multidiciplinary management of the rare tumor young adult a cooperative study of the GFS-GETO, Rare cancer network, GETTEc/REFCOR and SFCE. Ann Oncol. 2013;24:824-31.

5. Flechter CD, Bridge JA, Hogendoorm PC, Mertens F. WHO Classification of Tumord of Tissue and Bone. $4^{\text {th }}$ ed. Lyon: JRC Press; 2013.

6. Mirabello L, Zhu B, Koster R, Karlins E, Dean M, Yeager M, et al. Frecuency of pathogenic germline variants in cancer susceptibility genes in patients with osteosarcoma. JAMA Oncol. 2020;6:724-34.

7. Miller CW, Aslo A, Won A, Tan M, Lampkin B, Koeffler HP. alterations in p53 and MDM2 genes in osteosarcoma. J Cancer Res Clin Oncol. 1996;122:559-65.

8. Bouaoud J, Beinse G, Epaillard N, Amor-Sehlil M, Bidault F, Brocheriou I, et al. Lack of efficacy of neoadyuvant chemotherapy in adults patients with maxilofacial high grade osteosarcoma: a French experience in two reference centers. Oral Oncol. 2019;19:79-86.

9. Bosquet G, Janin A. Patient derived xenograft: an adyuvant technology for the treatment of mrtastatic disease. Pathobiol J Immunopathol Mol Cell Biol. 2016:83:170-6.

10. Asioli S, Righi A, Rucci P, Tarsitano A, Marchetti C, Bacchini P, et al p16 protein expresión and correlation with clinical and pathological features in osteosarcoma of the jaws: experience of 37 cases. Head Neck. 2017;39:1825-31.

11. Heymann MF, Lézot F, Heymann D. The contribution of immune infiltrates and the local microinveronmet in the pathogenesis of osteosarcoma. Cell Immunol. 2019;343:103-9.

12. Cortini M, Avnet S, Baldini N. Mesenchimal stroma: role in osteosarcoma progresión. Cancer Lett. 2017;405:90-9.

13. Brown HK, Schiavone K, Gouin F, Heymann MF, Heymann D. Biology of bone sarcomas and new therapeutic development. Calcific Tissue Int 2018; 102:174-95

14. Alvarez C, Monasterio G, Cavalla F, Córdova LA, Hernández M, Heymann $\mathrm{D}$, et al. Osteoimmunology of oral and maxilofacial diseases: translational applications based on biological mechanism. Front Immunol. 2019;10:1664.

15. Jawad SN, Abdullah BH. Proliferative apoptotic and angiogenic potentia in jaws and long bones osteosarcomas: a comparative immunihistochemical study. J Oral Pathol Med 2010;39:681-6.
16. Fuch B, Pritchard DJ. Etiology of osteosarcoma. Clin Orthop 2002; $40: 5240-52$.

17. Neville BW, Damm DD, Allen CM, Chi A. Oral and Maxillofacial Pathology. $3^{\text {rd }}$ ed. St Louis; Mosby, Elsevier; 2013.

18. Kondamari PS, Ramadas R, Cherian E, Thayalan D. Chondroblastic and fibroblas osteosarcoma of the jaws: report of two cases and review of literatura. Indian J Dent Res 2017;28:100-4.

19. Paparella ML, Olvi LG, Brandizzi D, Keszler A, Santini-Araujo E, Cabrini RL. Osteosarcoma of the jaw: an análisis a serie of 74 cases. Histopathology. 2013;63:551-7.

20. Mayordomo $E$, Machado I, Giner F Kresse SH, Myklebost O, Carda C et al. A tissue microarray study of osteosarcoma histopatology and immunohistochemical validation of xenotransplantaded tumor as preclinical models. Appl Immunihistochem Mol Morphol. 2010;18:453-61.

21. EnnekingWF. A system of staging musculoskeletal neoplasm. Clin Orthop Rel Res. 1986;204:9-24.

22. Laskar S, Basu A, Muckden MA, D'Cruz A, Pai S, Jambhekar N, et al. Osteosarcoma of the head and neck región: lessons learned from a single institution experience of 50 patients. Head Neck. 2008;30:1020-6.

23. Yildiz FR, Avei LG, Dereci O, Erol B, Celasun B, Gunhan O. Gnathic osteosarcoma experience of four institutions from Turkey. Int J Clin Exp Pathol. 2014;7:2800-8

24. Canadian Society of Otolaringology Head and Neck Oncology Study Group. Osteogenic Sarcoma of the mandible and maxilla: a Canadian review (1980-2000). J Otolaringol. 2004;33:139-44.

25. Ace B. The microscopic grading of cancer. In. Pack GT, Lwington EM, editors. Treatment of Cancer and Allied Disorders. New York: Paul B, Hoeber; 1946. p. 19

26. Unni KK, Dahlin DC. Grading of bone tumors. Sem Diag Pathol. 1984; $1: 165-72$.

27. Agrawal R, Bhavthankar JD, Mandale MS, Patil PP. Osteosarcoma of jaw with varing histomorphologic patterns: a case report. J Orthop Cas Rep. 2017;7:61-4.

28. Fernàndez R, Nikitakis NG, Pazoki A, Ord RA. Osteogenic sarcoma of the jaw: a 10 years experience. J Oral Maxiillofac Surg. 2017;65:1286-91.

29. Delgado R, Maafs E, Alfeiran A, Mohar A, Barrera JL, Zinser J, et al. Osteosarcoma of the jaw. Head Neck 1994;16:246-52.

30. Kassir RR, Rassekh CH, Kinsella JB, Segas J, Carrau RL, Hokanson JA Osteosarcoma of the had and neck: meta-analysis of nonrandomized studies. Laringoscope. 1997;107:56-61.

31. Mardinger O, Givol N, Talmi YP, Taicher S. Oteosarcoma of the jaw the chaim sheba medical center experience. Oral Surg Oral Med Oral Pathol Oral Radiol Endocr. 2001;91:445-51.

32. Velazco JA, Ladino JD, Garcia LF, Rojas CA, de León Rodríguez O, Bermudez L. Osteosarcoma mandibular fibroblástico de bajo grado: reporte de caso y revisión de la literatura. Univ Med. 2016:57:524-30.

33. Shetty SS, Dahaka RN, Venkadasalapathi N, Radhakrishnan R. Giant cell rich osteosarcoma of the jaw: a rare entity and review of literatura. J Oral Maxillofac Surg Med Pathol 2018;30:301-5.

34. Al Qathan CD, Alshedd M, Al Sablhan R. Epithelioid osteosarcoma the maxilla: a rare case report and review of literatura. Int J Surg Pathol. 2015;23:495-9.

35. Uma K,Cherian G, Patil SH. Small cell osteosarcoma of the mandible: a rare case report and review of its diagnostic aspecto. J Oral Maxillo Pathol. 2011;15:330-6. 